STRUCTURAL BIOLOGY COMMUNICATIONS

ISSN 2053-230X

Keywords: editorial; crystallization

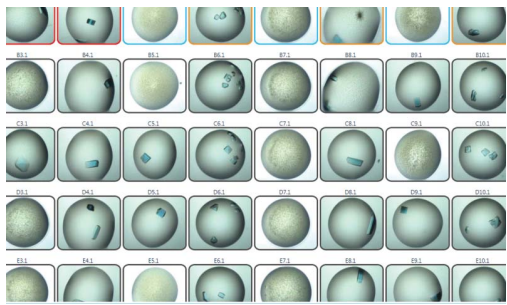

(C) 2019 International Union of Crystallography

\section{Welcoming Janet Newman with a BLAST on crystallization strategy}

\author{
Mark J. van Raaij*
}

Department of Macromolecular Structure, Centro Nacional de Biotecnologia, Consejo Superior de Investigaciones Cientificas, E-28049 Madrid, Spain. *Correspondence e-mail: mjvanraaij@cnb.csic.es

With this Editorial I would like to welcome Janet Newman as Section Editor of Acta Crystallographica F, Structural Biology Communications. Janet is a Principal Scientist at the Commonwealth Scientific and Industrial Research Organization (CSIRO) in Parkville, Victoria, Australia; Director of the Collaborative Crystallisation Centre (C3) and an experienced all-round crystallographer, with particular expertise in the difficult problem of macromolecular crystallization. She has published many papers in IUCr journals - the current count being 13 in Acta Cryst. D and 20 in Acta Cryst. F.

In this March 2019 issue, Janet has teamed up with Gabi Abrahams, an undergraduate student from the Undergraduate Research Opportunity Program at Melbourne University, to contribute an analysis of successful crystallization conditions for proteins that share sequence homology (Abrahams \& Newman, 2019). They find that crystallization conditions for proteins with a similar, but not identical, sequence are no more related to each other than those of completely different proteins. This is an important finding, contradicting common thought, and is perhaps disappointing for many researchers, because even for homologous proteins a completely new search in crystallization space will have to be performed. However, it is perhaps not that surprising because crystallization depends on stable crystal contacts formed by surface features of the protein, and it is these surface features that are most dissimilar between even quite similar proteins. In principle, if a protein structure were known, it should be possible to computationally predict a suitable crystal packing for it and suggest compounds that might promote the corresponding crystal contacts, but of course, the whole point of trying to crystallize a protein is that we don't know the structure!

On a positive note, it also means that if one has performed extensive unsuccessful crystallization trials for a given protein, switching to a homologous protein from a different species may lead to success. This strategy has been used before and this work provides an extra basis for it. Apart from the new protein potentially being more stable, and less flexible, it is unlikely to behave in the same way in crystallization space, so reusing the same conditions that were tried unsuccessfully for the related protein may now lead to well diffracting crystals.

I look forward to a fruitful collaboration with Janet in maintaining the excellent standards of publication of Acta Cryst. F, improving the quantity and the quality of the manuscripts we publish, and making Acta Cryst. F a venue of choice for submitting short but excellent Structural Biology Communications.

\section{References}

Abrahams, G. J. \& Newman, J. (2019). Acta Cryst. F75, 184-192. 